and not all are diagnostically specific. This lecture will attempt to review the role of genetic studies in SBCLs, highlighting their advantages and limitations.

\section{B-CELLS BEHAVING BADLY II: A BETTER BASIS TO BEHOLD BELLIGERENCE IN AGGRESSIVE B-CELL LYMPHOMAS}

\author{
Adam Bagg \\ Department of Pathology and Laboratory Medicine, University \\ of Pennsylvania, Philadelphia, PA, USA
}

Aggressive B-cell lymphomas include diffuse large B-cell lymphoma (DLBCL), the most common type of lymphoma encountered in most countries. Despite the single term, DLBCLs are a rather diverse group of lymphomas that can be subclassified using a variety of different approaches, including anatomical site, morphology, immunophenotype, and a number of genetic strategies. Nascent molecular technologies have led to the discovery of many of the genetic events underlying the pathogenesis of this group of diseases. Whether by defining gene signatures that subclassify DLBCL into subgroups based upon cell-of-origin, through dysregulation of key cellular pathways, or specific mutations, we are approaching an era in which personalised diagnostics, prognostication and therapy are imminent. Attempts to develop surrogate immunohistochemical algorithms for some of these genetic phenomena have been variably successful. This lecture will focus on DLBCL and highlight one particular shade of so-called grey zone lymphoma and its relationship to double-hit lymphomas. An attempt will also be made to synthesise the rational use of genetic testing of lymphomas covered in both this and the preceding lecture on small B-cell neoplasms.

\section{TESTS FOR TTP/aHUS}

\section{Theo de Malmanche}

Pathology North (Hunter) Immunology, Newcastle, NSW, Australia

Thrombotic thrombocytopenic purpura (TTP) follows an excess of ultra large multimers of von Willebrand factor (vWF), which follows reduced serum ADAMTS13 activity. Microangiopathy which follows intoxication with Shiga toxins, e.g., some $E$. coli (STEC) are perhaps best labelled as STEC-HUS. ADAMTS13 activity and stool STEC toxin are specific assays for these diseases.

Many STEC negative/ADAMTS13 replete cases are complement mediated. Organ damage will occur without affecting routine complement assays. Triggering events are minor and not specific for microangiopathy (e.g., rotavirus). Testing aims to demonstrate (1) the predisposing tendency to complementmediated microangiopathy (detected in $\sim 30 \%$ of cases) or (2) complement activation (largely anecdotal and case studies).

Abnormalities of regulators of complement activation (RCA) molecules have the highest yield, specifically, complement factors $\mathrm{H}$ and $\mathrm{I}(\mathrm{CFH}, \mathrm{CFI})$, and membrane cofactor protein $(\mathrm{MCP}$, CD46. Complement component 3 (C3), complement factor B, thrombomodulin have a lower yield.

Complement activation can be demonstrated with serum levels of terminal complement complex (TCC, aka sMAC, or sC5b-9) and biopsies for vascular deposition of the same TCC/MAC (either involved organ or random skin biopsies).

Reference

1. Nester C, Barbour T, de Cordoba SR, et al. Atypical aHUS: state of the art. Mol Immunol 2015; 67: 31-42.

\section{AN UPDATE ON TTP INCLUDING DATA FROM THE AUSTRALIAN TTP/TMA REGISTRY}

\section{P. Blombery \\ Peter MacCallum Cancer Centre and Monash University, Melbourne, Vic, Australia}

Thrombotic thrombocytopenic purpura (TTP) is a life-threatening thrombotic microangiopathy (TMA) which is the result of a severe deficiency of ADAMTS13 activity. In the majority of cases, the ADAMTS13 deficiency is the result of auto-antibodies produced against ADAMTS13 which impair protein activity. With the recognition and characterisation of the pathological basis of acquired TTP has come rational therapies including more targeted and less toxic immunosuppression (e.g. rituximab), targeted agents against the vWF platelet interaction (caplacizumab) and recombinant ADAMTS13.

The Australian TTP/TMA registry was established to collect data on Australian patients with all TMAs with the aim of creating a resource to help assess the patterns of diagnosis and management of this group of disorders in Australia. The registry is maintained by the Monash University Department of Epidemiology and Preventive Medicine (DEPM) under the auspices of the Transfusion Outcomes Research Collaboration, a partnership with the Australian Red Cross Blood Service (ARCBS), and began collecting data in 2009. Analysis of data from patients with TTP in the TTP/TMA registry has given insights into the current state of diagnosis and treatment of TTP in Australian patients and identified potential areas for practice improvement which will be shared in this presentation.

\section{aHUS IN CHILDREN: A SEVERE DISEASE WITH INCREASINGLY BETTER DIAGNOSTIC AND TREATMENT OPTIONS}

Stephen Alexander

Children's Hospital at Westmead, NSW, Australia

Atypical haemolytic uraemic syndrome (aHUS) accounts for around $10 \%$ of children presenting with haemolytic uremic syndrome, a triad of thrombocytopenia, haemolytic anaemia and renal failure. As opposed to diarrhoeal causes usually associated with $E$. coli infection producing the toxin STEC, aHUS generally presents without diarrhoea and is frequently associated with defects in the complement pathway leading to over-activation of complement.

The major cause of paediatric aHUS is due to defects in complement factor $\mathrm{H}$, a regulatory protein that limits complement activation. Mutations in $\mathrm{CFH}$ that are both dominant and recessive have been described. There are now over 10 other genes that have been described in aHUS with around $40 \%$ of sporadic cases of aHUS without an identified genetic cause.

Clinically patients often present acutely with an infectious prodrome, and may require supportive haemodialysis and transfusion. Children occasionally have extra renal manifestations 